\title{
Light Detection in Open-circuit Voltage Mode of Organic Photodetectors
}

Marcin Kielar ${ }^{*}$, Tasnuva Hamid, Martin Wiemer, François Windels, Lionel Hirsch", Pankaj Sah and Ajay K. Pandey

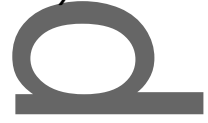

Dr. M. Kielar, Dr. F. Windels, Prof. P. Sah

Queensland Brain Institute, The University of Queensland

St Lucia, QLD 4072, Australia

E-mail:m.kiełar@uq.edu.au

Dr. M. Kielar, T. Hamid, Dr. A. K. Pandey

Robotics and Autonomous Systems, School of Electrical Engineering and Computer Science, Science and Engineering Faculty, Queensland University of Technology

Brisbane, QLD 4001, Australia

E-mail:a2.pandey@qut.edu.au

Dr. M. Wiemer, Or. L. Hirsch

Univ. Bordeaux, IMS, CNRS, UMR 5218, Bordeaux INP, ENSCBP

F-33405 Talence, France

E-mail: lionel.hirsch@ims-bordeaux.fr

Keywords: organic photodetectors, open-circuit voltage, photovoltage, shunt resistance

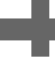

Organic photodetectors (OPDs) are promising candidates for next-generation light sensors as they combine unique material properties with high-level performance in converting photons into current. However, low-level light detection is often limited by device dark current. Here, we show that the

This is the author manuscript accepted for publication and has undergone full peer review but has not been through the copyediting, typesetting, pagination and proofreading process, which may lead to differences between this version and the Version of Record. Please cite this article as doi: 10.1002/adfm.201907964. 
open-circuit voltage $\left(V_{o c}\right)$ regime of OPDs is efficient for detecting low light signals $\left.(<100 \mu \mathrm{W} \mathrm{cm})^{-2}\right)$. We show that the light-dependence of $V_{o c}$ exhibits two distinct regimes as function of irradiance: linear and logarithmic. Whereas the observed logarithmic regime is well understood in organic photovoltaic cells (OPVs), we show experimentally and theoretically that the linear regime is due to the non-infinite shunt resistance of the device. Overall, OPDs composed of rubrene and fullerene show photovoltage light sensitivity across 9 orders of magnitude with a detection limit as low as 400 $\mathrm{pW} \mathrm{cm}{ }^{-2}$. A photovoltage responsivity of $1.75 \mathrm{~V} \mathrm{~m}^{2} \mathrm{~W}^{-1}$ demonstrates the highly efficient performance without the necessity to correct high dark current. This approach opens up new possibilities for resolving low light signals and provides simplified design rules for OPDs.

\section{$(3$}

\section{Introduction}

The extensive research on $\pi$-conjugated materials over the last two decades has provided a better understanding of the light-harvesting mechanisms in optoelectronic devices. ${ }^{[1-5]}$ Consequently, organic photodetectors (OPDs) show not only excellent quantum efficiency, ${ }^{[6-8]}$ and tunable spectral selectivity, ${ }^{9}{ }^{11]}$ but can also take advantage of photon up-conversion, ${ }^{[12,13]}$ and photomultiplication mechanisms. ${ }^{[7,14]}$ Most importantly, organic semiconductor-based sensors have closely approached the performance of conventional inorganic photodetectors demonstrating remarkable levels of responsivity, dark current and even long-term stability. ${ }^{[15,16]}$ It is thus no surprise that OPDs are widely thought to underpin the next generation of digital imaging technology, garnering strong interest from both academia and industry. As a matter of fact, research on OPDs and related development efforts have been growing exponentially (Figure S1, Supporting Information).

To achieve ideal performance, OPDs usually operate in the photoconductive mode, where an external reverse bias is applied to improve the charge carrier collection efficiency. ${ }^{[17]}$ The resulting photocurrent varies linearly with the light intensity across several orders of magnitude. ${ }^{[8]} \mathrm{A}$ major disadvantage of the reverse bias mode is the rising dark current, i.e., the leakage current that flows across the device in the absence of light. Suppressing dark current, as desired for resolving low light intensities, usually requires highly engineered device architectures. This is frequently achieved by introducing additional interlayers into the device structure. ${ }^{[16,18,19]}$ Furthermore, detection of photocurrent in the picoampere range or below involves the use of shielded, high precision detection methods involving source-measure units, lock-in amplifiers or spectrum analyzers. ${ }^{[20-22]}$

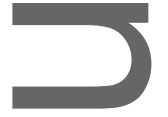

Here we present an alternative, easy-to-handle approach for low-light detection with OPDs. We demonstrate that using basic-structure OPDs, it is possible to accurately sense light intensity through changes in open-circuit voltage $\left(V_{o c}\right)$. For illustration, we investigate the performance of a rubrene/fullerene bilayer photodiode in both photoconductive and photovoltage modes. We first consider the photocurrent response of our example OPD to various irradiance levels and voltage

This article is protected by copyright. All rights reserved. 
biases including short-circuit condition. Then, we turn towards $V_{o c}$ regime as our new figure of interest. We present the photovoltage response to increasing irradiance and demonstrate that it can be separated into a linear regime at low, and a logarithmic regime at high intensity levels. We characterize the linear regime in terms of linear dynamic range and responsivity of $1.75 \mathrm{~V} \mathrm{~m}^{2} \mathrm{~W}^{-1}$. Thanks to high voltage responsivity, we find that $V_{o c}$ signal remains strong even at very low light intensities and can be measured using a simple multimeter. The association of the linear regime in photovoltage mode with shunt resistance of OPD is then discussed. Finally, we examine the behavior of our OPD under open-circuit conditions to pulsed illumination at various frequencies. We demonstrate that OPDs operating in $V_{\text {oc }}$ mode are able to sense irradiance as low as $400 \mathrm{pW} \mathrm{cm}$. We conclude our investigation with a discussion on strategies for performance improvements and possible applications.

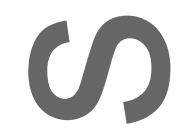

\section{Results and Discussion}

\subsection{Rubrene and fullerene for high performance OPDs}

For low-light signal detection using $V_{o c}$, we used well-known and extensively studied materials in the field of organic electronics. Indium tin oxide (ITO) is used as transparent electrode, PEDOT:PSS serves as hole transport interlayer, rubrene as electron donor, fullerene $\left(C_{60}\right)$ as electron acceptor, barium ( $\mathrm{Ba}$ ) and silver ( $\mathrm{Ag}$ ) as top electrode. The choice of the active layer composed of rubrene and $\mathrm{C}_{60}$ is driven by its universality. One can use rubrene and $\mathrm{C}_{60}$ in organic photovoltaic cells that specifically develop a large $V_{o c}$ of about $940 \mathrm{mV}$ at 1 sun. ${ }^{[23]}$ These two materials can also be used in organic light-emitting diodes (with yellow electroluminescence at sub-band gap turn-on voltage), ${ }^{[24]}$ or in multifunctional diodes, as described in our recent study. ${ }^{[25]}$ The device structure is shown in Figure 1a. Chemical structures of the materials, the energy level diagram and the working principle of the OPD, can be found in Figure S2 and S3.

a)

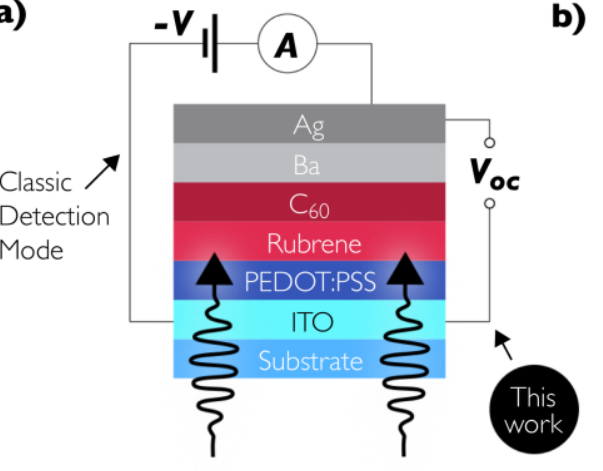

b)

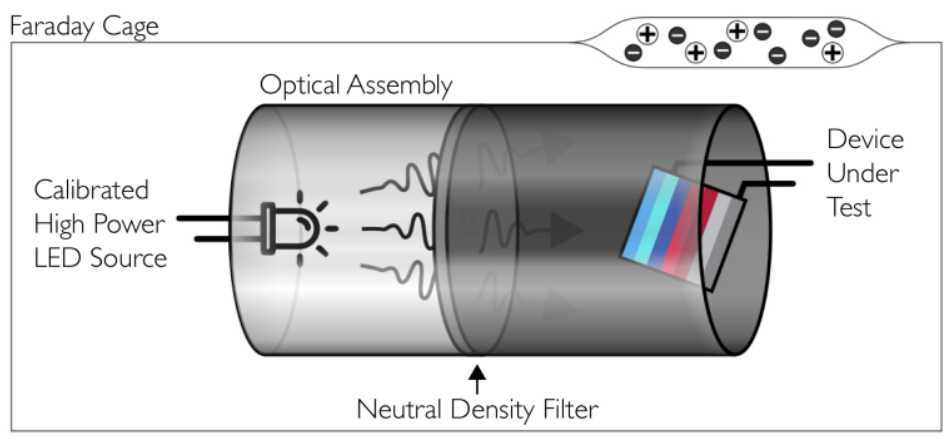

Figure 1. Device structure and experimental set-up for low-level light detection. a) Cross-sectional device structure of the photodetectors studied in this work. The classic photodetection mode (where a negative bias is applied and a resulting photocurrent is recorded) and the proposed photodetection mode via changes in open-circuit voltage $\left(V_{o c}\right)$ are shown. Undulating arrows represent incident photons (here $536 \mathrm{~nm}$ ). b) Cartoon showing the main experimental set-up: a high-power green LED light source capable of delivering irradiance levels over 6 orders of magnitude is inserted into the optical assembly containing a neutral density (ND) filter. The reduced light intensity strikes the organic

This article is protected by copyright. All rights reserved. 
photodetector placed at the other end of the optical assembly. The ensemble is placed inside a double-shielded Faraday cage.

To sense optoelectronic performance of OPD devices, an optical assembly featuring a green LED and a set of heutral density (ND) filters was designed and built from scratch. A schematic illustration of the experimental set-up is shown in Figure 1b. The high-power $536 \mathrm{~nm}$ LED (emission spectrum shown in Figure S4) was calibrated to deliver light intensities from $1 \mathrm{nW} \mathrm{cm}$ to $25 \mathrm{~mW} \mathrm{~cm}^{-2}$ (calibration curve in Figure S5). These irradiance levels are further reduced by inserting a ND filter into the optical assembly. Two filters featuring the optical densities (OD) of 2.0 and 5.0 were used to reduce incident irradiance approximately by a factor of $10^{2}$ and $10^{5}$, respectively (Figure S6 and S7). The lowest accessible irradiance was $10 \mathrm{fW} \mathrm{cm}^{-2}$ and is near the limit of detection of the human eye. ${ }^{[26]}$ We note here than weaker light intensities can be achieved by combining both ND filters together (OD 7.0), and the resulting light can be visualized through a commercial CMOS sensor (Figure S8 and S9). Finally, the OPD was placed at the other end of the optical assembly and kept at $50 \mathrm{~mm}$ from the LED for all measurements. To eliminate human and environmental electromagnetic noise, the proposed set-up was placed inside a custom built, double-shielded Faraday cage (Figure S10 and S11) and all measurements were performed remotely.

\subsection{Conventional light sensing with OPDs}

Figure 2 a shows the current density-voltage $(\mathrm{J}-\mathrm{V})$ characteristics of the fabricated organic photodetector in the dark and under monochromatic (536 nm) irradiance levels of 0.01, 0.1, 1 and $10 \mathrm{~mW} \mathrm{~cm}^{-2}$. As expected, the photocurrent is proportional to the light intensity under reverse bias, i.e., it increases by a factor of 10 for each consecutive irradiance. One can also note that for all light intensities, and with regards to increasing applied bias, a rise in the photocurrent response can be observed as well. At $1 \mathrm{~mW} \mathrm{~cm}^{-2}$, we observe a photocurrent increase of $220 \%$ between 0 and -2 $\mathrm{V}$. The external quantum efficiency $(E Q E)$ measurements, shown in Figure $2 \mathrm{~b}$, confirm high dependence of the device performance on the applied voltage. The highest EQE values are measured at $495 \mathrm{~nm}$, which corresponds to one of three well-resolved vibronic peaks in the absorption spectrum of rubrene (the other two peaks, 462 and $528 \mathrm{~nm}$, can also be seen). ${ }^{[27]}$ The photocurrent responsivity $\left(\mathcal{R}_{I}\right)$ at $0 \mathrm{~V}$ (calculated using $E Q E$ at $536 \mathrm{~nm}$ ) is $63 \mathrm{~mA} \mathrm{~W}^{-1}$ and scales to 99 $\mathrm{mA} \mathrm{W} \mathrm{W}^{-1}$ at $-1 \mathrm{~V}$. The highest value of $136 \mathrm{~mA} \mathrm{~W}^{-1}$ is reached at $-2 \mathrm{~V}$. These responsivities are in good agreement with the J-V characteristics, shown in Figure 2a, and reflect the general need for OPDs to operate under reverse bias.

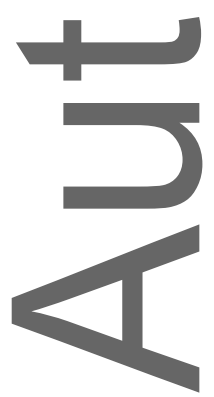

This article is protected by copyright. All rights reserved. 

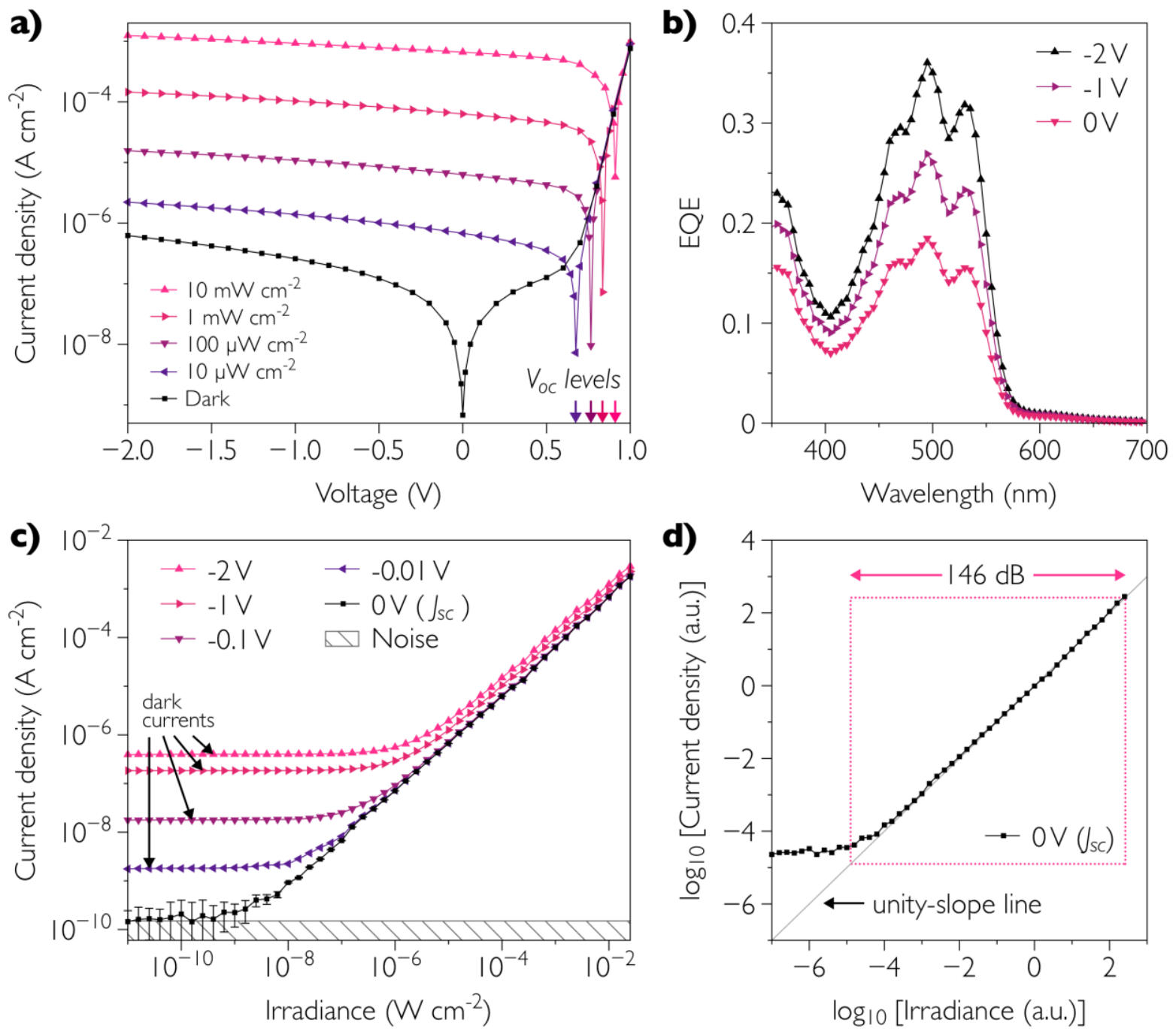

Figure 2. Performance of OPDs in the conventional photocurrent mode. a) Extended current density-voltage (J-V) characteristics of the fabricated device in dark and under different irradiances up to $10 \mathrm{~mW} \mathrm{~cm} \mathrm{c}^{-2}$. A calibrated, green LED $(536 \mathrm{~nm})$ is used as light source. b) External quantum efficiency of the fabricated device at $0,-1$ and $-2 \mathrm{~V}$. c) Photocurrent density as a function of incident irradiance for the photodetector under short-circuit condition $\left(J_{s c}, 0 \mathrm{~V}\right)$ and under various negative voltages. The corresponding dark current levels are shown. $d$ ) Linear dynamic range of the OPD operating in short-circuit condition and under irradiance levels between $10 \mathrm{pW} \mathrm{cm}^{-2}$ and $25 \mathrm{~mW} \mathrm{~cm}^{-2}$.

Unfortunately, increasing reverse bias to enhance the OPD responsivity comes at a significant cost. From the $J-V$ measurement in the dark (black curve in Figure 2a), one can note that dark current becomes higher with increasing reverse bias. We measured $-23,-259$ and $-623 \mathrm{nA} \mathrm{cm} \mathrm{cm}^{-2}$ at $-0.1,-1$ and $-2 \mathrm{~V}$, respectively. High dark currents likely reflect a lack of hole/electron blocking interlayers in the device structure. Since the photodetector dark current adds up to the signal under illumination, it can saturate the photocurrent at low light intensities. In our case, dark current dominates the photogenerated light current at $2.6 \mu \mathrm{W} \mathrm{cm}$ under $-1 \mathrm{~V}$. At $-2 \mathrm{~V}$, dark current overshadows light signals for irradiance levels below $4.6 \mu \mathrm{W} \mathrm{cm}$. One could still try to resolve the sub-dark current signal by simply subtracting the dark current contribution from the photocurrent. However, in most cases frequency-dependent lock-in amplifiers or spectrum analyzers are required. Alternative and simple ways of low-light detection are therefore highly desired.

This article is protected by copyright. All rights reserved. 
Keeping in mind that dark current becomes dominant at low light intensities, we investigated its influence on the lowest detection limit. For clarity, the lowest detection limit in our experiments is defined as the weakest light intensity that gives a significant ( $95 \%$ of confidence) difference in photocurrent with respect to the measurement in the dark. Figure $2 c$ shows the linearity measurements where the photocurrent density of the OPD was measured as a function of incident irradiance. As it can be seen, an increase in negative bias applied to the photodetector results in a reduction of linear detection range. This establishes the direct influence of dark current in oversaturating the OPD signal at low light intensities. According to Figure S12, a light intensity of 2.4 $\mathrm{nW} \mathrm{cm}{ }^{-2}$ is the limit of detection at $-0.01 \mathrm{~V}$. This value rises up to $12.6 \mathrm{nW} \mathrm{cm}$ for the same device biased at $-2 \mathrm{~V}$. We note here that the lowest irradiance detectable at $0 \mathrm{~V}, 1.4 \mathrm{nW} \mathrm{cm}^{-2}$, is underestimated since it is limited by the resolution of the experimental set-up. Using lock-in amplifier, researchers have claimed light intensity detection as weak as $7.6 \mathrm{pW} \mathrm{cm}{ }^{-2}$ under shortcircuit current mode of OPD operation. ${ }^{[21]}$

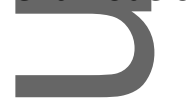

As discussed above, the dark current not only restricts the detection limit but it also affects the linear dynamic range $(L D R)$, i.e., the range of linear responsivity. Figure $2 \mathrm{~d}$ shows the double logarithmic plot of photocurrent versus irradiance where the slope is unity, for the device operating in short-circuit condition (highest linear range). The $L D R$ of $146 \mathrm{~dB}$ is observed. This value decreases to 122 and $70 \mathrm{~dB}$ at -0.01 and $-1 \mathrm{~V}$, respectively. These values demonstrate that the higher the applied reverse bias, the lower the $L D R$. Since the responsivity increases with reverse bias, our measurements also indicate that a large range of linear responsivity can only come at the sacrifice of high responsivity. New photosensing approaches are thus needed to unlock the limited performance of the OPD in order to deliver simultaneously excellent responsivity and linearity.

\subsection{Photosensing in the open-circuit voltage mode of OPDs}

Having demonstrated the limitations of photoconductive mode in OPDs, we now turn to another observable, the open-circuit voltage, and investigate its potential in photosensing with a basic-structure OPD, deprived of electron and hole blocking interlayers. To the best of our knowledge, ho research study has investigated the use of $V_{o c}$ for light detection in organic photodetectors, especially under low-light conditions. More generally, the use of $V_{o c}$ and its dependence on light intensity are largely neglected in research studies on OPDs. The open-circuit voltage is however one of the key parameters of organic photovoltaic cells (OPVs), its origin and role in OPV performance have been extensively investigated. ${ }^{[17,28]}$ That being said, OPVs operate at high light intensities $\left(\sim 100 \mathrm{~mW} \mathrm{~cm}^{-2}\right)$ whereas OPDs tend to function under low irradiance to minimize overall power consumption and degradation mechanisms (such as photooxidation). ${ }^{[29]}$ It is worth emphasizing that the $V_{o c}$ mode could actually lead to a self-powered detector.

This article is protected by copyright. All rights reserved. 
To unveil the light dependent nature of the $V_{o c}$ and its role in low-level light detection, we monitored the $V_{o c}$ response of the rubrene/fullerene OPD as a function of incident irradiance. The result can be seen in Figure 3a. One can note that the $V_{o c}$ signal varies linearly with irradiance for light intensities weaker than $25 \mu \mathrm{W} \mathrm{cm}$ (coefficient of determination $R^{2}=0.998$ ). Then, a transition from linear to logarithmic regime occurs for light intensities between 25 and $100 \mu \mathrm{W} \mathrm{cm}{ }^{-2}$. And finally, a logarithmic regime for the $V_{o c}$ signal is observed under higher irradiances (with $R^{2}=0.966$ ). Both regimes are detailed in Figure S13.
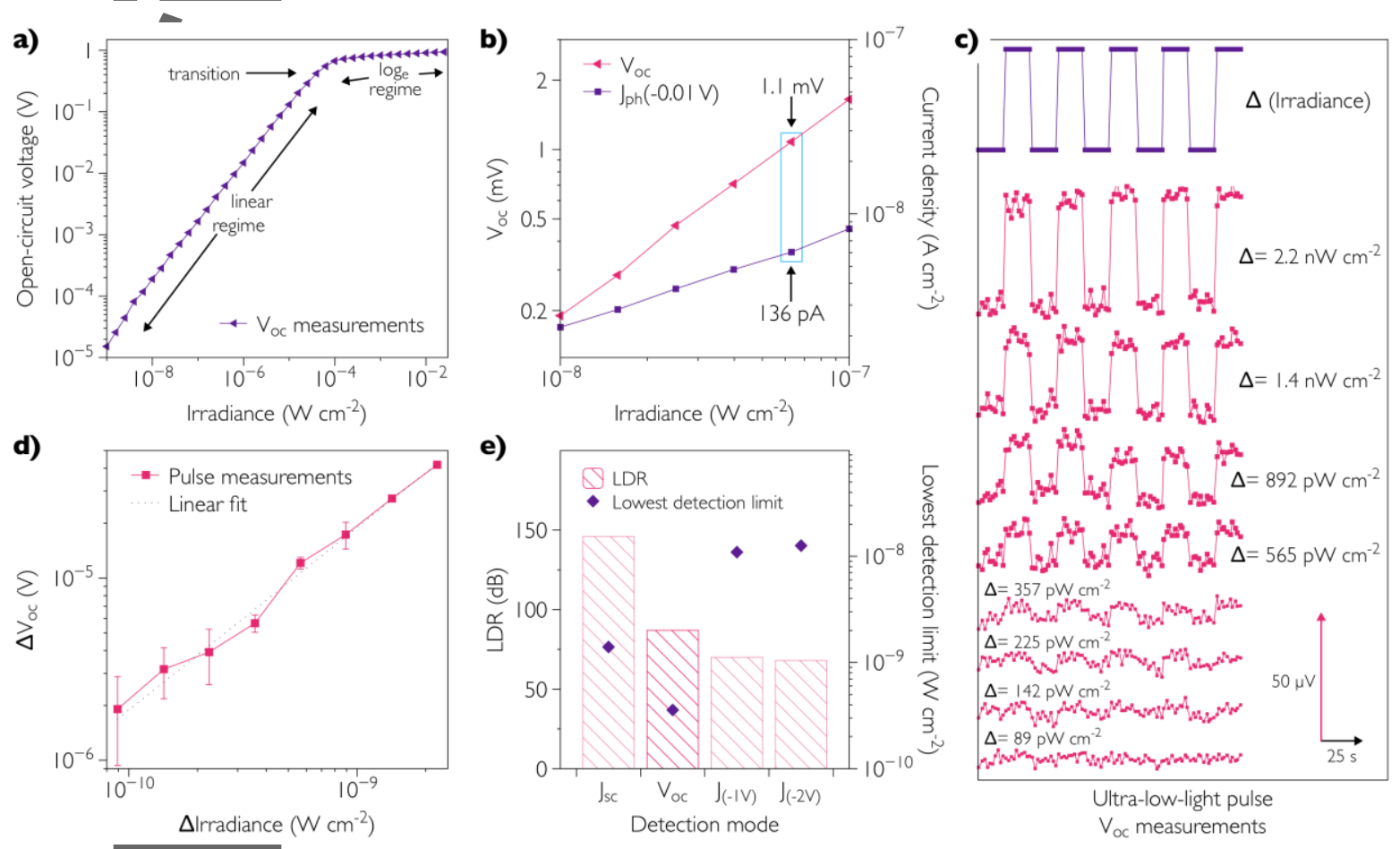

Figure 3. Light detection through changes in open-circuit voltage in organic photodetectors. a) $V_{o c}$ signal as a function of irradiance. Both linear and logarithmic regimes are highlighted. b) Comparison of the $V_{o c}$ and the photocurrent signals at the same irradiance and for the same photodetector. $c)$ Voltage $\left(V_{o c}\right)$ response of the fabricated device to the low intensity pulses of green $(536 \mathrm{~nm})$ light. ND filters are used here to produce irradiance levels from $89 \mathrm{pW} \mathrm{cm} \mathrm{cm}^{-2}$ to $2.2 \mathrm{nW}$ $\mathrm{cm}^{-2}$. d) $\mathrm{V}_{\mathrm{oc}}$ signal as a function of irradiance. Error bars show the standard deviation. e) Summary of the performance of the detector in the $V_{o c}$ and photocurrent modes under various biases. LDR and the lowest detection limit are shown.

The linear response of $V_{o c}$ with incident light suggests a definition of a new figure of merit analogous to the responsivity in the photocurrent mode $\left(\mathcal{R}_{I}\right)$ that is expressed in $\mathrm{A} \mathrm{W}^{-1}$ :

$\mathcal{R}_{I}=\frac{\text { Photocurrent } I}{\text { Ineident light power }}$

However, a small, but yet fundamental difference between $I$ and $V_{o c}$ must be taken into consideration here. Photocurrent, on the one hand, results from the total area-integrated light power converted into free charge carriers. Photovoltage, on the other hand, reflects the charge density-related difference in electrochemical potential, and is, hence, associated with the incident irradiance, i.e., the light power per area, and not the total light power. To illustrate this, one may think of two photodetectors that are different in size but otherwise identical. Under homogenous illumination, the absolutely identical local kinetics of charge carrier generation, recombination, and

This article is protected by copyright. All rights reserved. 


\section{WILEY-VCH}

extraction would yield exactly the same concentration profiles of charge carriers throughout the active layers, and thus the same photovoltage responses in both devices. Nevertheless, the smallerin-size photodetector would evidently capture less light in total, and thus also generate less photocurrent than its larger-in-size counterpart. For this reason, it is more convenient to define the photovoltage responsivity as follow:

$\mathcal{R}_{V}=\frac{\text { Photovoltage } V_{o c}}{\text { Incident irradiance }}$

with $\mathcal{R}_{V}$ expressed in $\mathrm{V} \mathrm{m}^{2} \mathrm{~W}^{-1}$. Following the definition of equation (2), the slope of the linear portion of the curve from Figure 3 a reveals a voltage responsivity of $1.75 \mathrm{~V} \mathrm{~m}^{2} \mathrm{~W}^{-1}$.

Figure $3 \mathrm{~b}$ illustrates the practical importance of a large voltage responsivity. An irradiance as low as $63 \mathrm{nW} \mathrm{cm}$ produces a strong $V_{o c}$ signal of about $1.1 \mathrm{mV}$ that can easily be detected with a simple digital laboratory multimeter. In contrast, the same irradiance would only raise the photocurrent to around $136 \mathrm{pA}$ (or $6 \mathrm{nA} \mathrm{cm}$ ), which would be beyond the range of the same multimeter. This high voltage responsivity gives thus an advantage over the photocurrent, especially at low light intensities. These results are particularly important in wake of the recent studies on photomultiplication thin film photodetectors where current responsivities greater than $1000 \mathrm{~A} \mathrm{~W}^{-1}$ have been reported. ${ }^{[14]}$ Another advantage of the photovoltage responsivity lies in its independence from the detector surface area, which is a direct implication of equation (2). This could, in principle, proof very effective in case of detector arrays composed of elements in various sizes, or with respect to miniaturization.

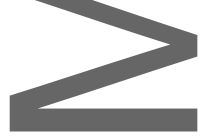

To determine the detection limit in $V_{o c}$ mode for the OPD, we performed ultra-low light pulse measurements. The pulse frequency was arbitrary set to $3.2 \mathrm{mHz}$ and the ND filters were used to produce relative irradiance levels down to $89 \mathrm{pW} \mathrm{cm}^{-2}$. From Figure $3 c$, one can note that the voltage output is strong even at low irradiances. For example, a $V_{\text {oc }}$ signal of $27 \pm 3 \mu \mathrm{V}$ is observed at $1.4 \mathrm{nW}$ $\mathrm{cm}^{-2}$. The signal further decreases with decreasing irradiance until reaching a point at which it becomes indistinguishable from the noise signal. The overall $V_{o c}$ dependence on ultra-low light is summarized in Figure 3d. A strong linear trend is observed $\left(R^{2}=0.997\right)$ and is consistent with the linear regime seen in Figure 3a. Therefore, the steady-state linearity measurement can be further extended with the pulsed measurements (see Figure S13). We found that the detection limit is as low as $400 \mathrm{pW} \mathrm{cm}{ }^{-2}$, as detailed in Figure S14. The fact that $V_{\text {OC }}$ mode of photodetection described here is able to record extremely low light signals, that too with using an OPD device of $\mathcal{R}_{\mathrm{I}}=0.06 \mathrm{~A} / \mathrm{W}$ with no lock-in amplifiers, shows the complementary nature and robustness of $V_{\text {oc }}$ mode for OPDs.

In addition to the detection limit in the $\mathrm{pW}$ range, the $V_{o c}$ mode is also found to provide a high LDR of $87 \mathrm{~dB}$ (Figure S15). The LDR is limited, from one side, by the lowest detection limit, and, from the other side, by the linear-to-logarithmic transition seen in Fig 3a. Interestingly, since photoconductive

This article is protected by copyright. All rights reserved. 


\section{WILEY-VCH}

mode shows a linear response at high irradiances where the $V_{o c}$ signal saturates into the logarithmic regime, one could selectively use the $V_{o c}$ or photocurrent modes of the same device to create dualmode, high-LDR organic photodetectors.

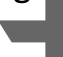

\subsection{Linear regime of the $V_{o c}$ dependence on irradiance}

Given the fact that a number of studies have shown, theoretically and experimentally, that the $V_{o c}$ varies logarithmically with illumination intensity, ${ }^{[28,30-34]}$ the physics behind the linear regime is yet to be understood. The transition between logarithmic and linear regimes has been observed in OPVs but it had not been described nor used for low-level light detection. ${ }^{[35]}$ Most $V_{o c}$ models are based on the work published in 2005 by Koster et al. that expresses the open-circuit voltage in terms of bimolecular recombination. ${ }^{[34]}$ In this model:

$V_{o c}=\frac{E_{g a p}}{q}-\frac{k_{B} T}{q} \ln \left(\frac{(1-P) \gamma N_{c}^{2}}{P G}\right)$

where $E_{g a p}$ is the band gap, $q$ is the elementary charge, $k_{B}$ is Boltzmann's constant, $T$ is temperature, $P$ is the dissociation probability of a bound electron-hole pair into free charge carriers, $\gamma$ is the Langevin recombination constant, $N_{c}$ is the effective density of states, and $G$ is the generation rate of bound electron-hole pairs. Since $G$ is the only parameter assumed to be proportional to the intensity, it is evident from equation (3) that $V_{o c}$ varies logarithmically with irradiance. It would be thus impossible to fit our data from Figure $3 a$ with equation (3).

A significant contribution to the model expressed in equation (3) has been made in 2015 by Proctor et al. who demonstrated that the leakage current (i.e., low shunt resistance) was not negligible in solar cells and should thus be taken into account in order to successfully match the experimental data. ${ }^{[36]}$ The equation (3) gains thus one additional term:

$V_{o c}=\frac{E_{g a p}}{q}-\frac{k_{B} T}{q} \ln \left(\frac{(1-P) \gamma N_{C}^{2}}{P G-V_{o c} / q L R_{S h}}\right)$

where $L$ is the active layer thickness, and $R_{s h}$ the shunt resistance. Since this model was developed to gain insights into the recombination processes in OPVs, the light intensities used by Proctor were close to what a solar simulator can produce (i.e., between 1 and $100 \mathrm{~mW} \mathrm{~cm}^{-2}$ ). At these irradiance levels, we also observe a logarithmic dependence (see Figure 3a) with a slope $S$ of 1.35 indicating contributions from mono- and bimolecular recombination terms. ${ }^{[37,38]}$ At first sight, the equation (4) cannot explain the linearity observed in Figure $3 a$ (and Figure 3d) and it cannot be easily solved for the open-circuit voltage either unless using numerical methods (variable $V_{o c}$ appearing on both sides). To address this issue, instead of solving $V_{o c}=f(G)$, we express the inverse equation $G=f\left(V_{o c}\right)$ as follow:

$G=\left[\frac{(1-P) \gamma N_{c}^{2}}{P} \exp \left(\frac{q V_{o c}-E_{g a p}}{k_{B} T}\right)\right]_{\text {exp.term }}+\left[\frac{V_{o c}}{q L P R_{S h}}\right]_{\text {lin.term }}$

This article is protected by copyright. All rights reserved. 
Two terms, exponential $\left(G \propto e^{V_{o c} / k_{B} T}\right)$, and linear $\left(G \propto V_{o c}\right)$, are distinguishable. From this, one can note that the first term drops exponentially with decreasing $G$ and practically vanishes for low $V_{o c}$ values $\left(V_{o c} \ll E_{g a p} / q\right.$ where $\left.E_{g a p} / q>1 V\right)$. If so, $G$ (and thus light intensity) varies linearity with $V_{o c}$ according to the second term. In other words, equation (4) predicts indeed the linear regime of the $V_{o c}$ on irradiance for low light intensities.
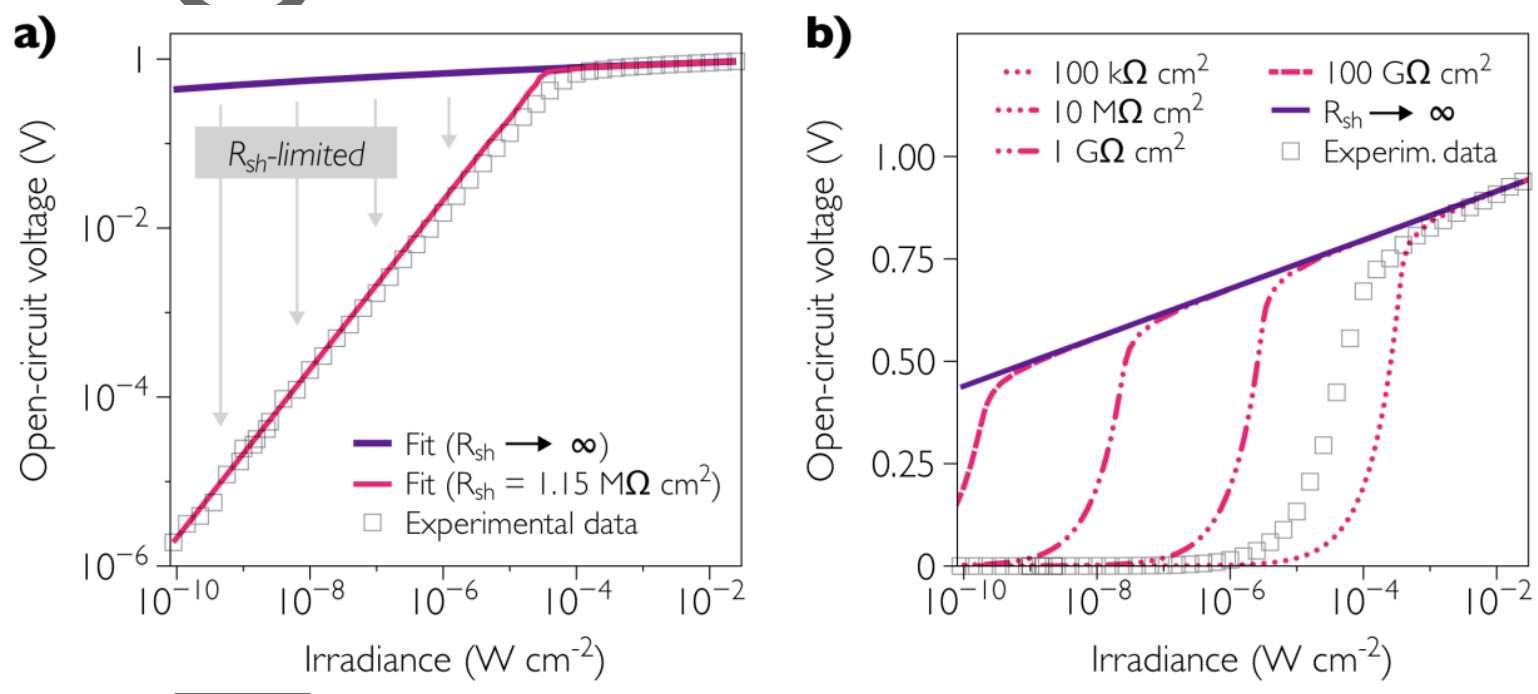

Figure 4. Modelling of the light intensity dependence of the $V_{o c}$. a) Fits to the experimental data using equation (3) where leakage current through $\boldsymbol{R}_{s h}$ is neglected (ideal device) and equation (4) that considers parasitic leakage currents (and thus low shunt resistance). b) Semi-log plot illustrating the relation between the device's shunt resistance and the shape of the light intensity dependence of the $V_{o c}$. Experimental data are plotted for a photodetector with $R_{s h}$ equal to $1.15 \mathrm{M} \Omega \mathrm{cm}^{2}$. Only an ideal device (no leakage current) would show a logarithmic dependence (purple straight line) across all irradiance levels in the considered range.

To illustrate the relation between the shunt resistance and the linear regime, we described our experimental data with the above model. Two fits, one based on equation (3) (zero leakage, purple line) and the second based on equation (4) (non-zero leakage, rose line) are shown in Figure 4a. The applied parameters are: $E_{g}=1.35 \mathrm{eV}, P=0.55, L=65 \mathrm{~nm}, N_{c}=1.2 \times 10^{25} \mathrm{~m}^{-3}, R_{s h}=1.15 \mathrm{M} \Omega \mathrm{cm}^{2}, \gamma=$ $6 \times 10^{17} \mathrm{~m}^{3} \mathrm{~s}^{-1}$. Generation rate of $2.7 \times 10^{27} \mathrm{~m}^{-3} \mathrm{~s}^{-1}$ is set to correspond to an illumination intensity of $80 \mathrm{~mW} \mathrm{~cm}{ }^{-2} \cdot{ }^{[34]}$ The shunt resistance is within the range measured for OPDs used in this study (see Figure S16). One can note that both regimes, linear and logarithmic, as seen in Figure 3a, can be explained with the modified model from Proctor et al. Interestingly, the shunt resistance significantly shapes the $V_{o c}$ signal at low light intensities (see the arrows in Figure 4a). Its effect on $V_{o c}$ is further explored in Figure $4 \mathrm{~b}$. One prediction of this model suggests that the higher the shunt resistance is, the larger the logarithmic regime would be (see Supplementary Video 1$)$. At the limit $\left(R_{s h} \rightarrow \infty\right.$, no leakage current), only a logarithmic regime is observed. In this case, equation (4) reduces to equation (3).

For the sake of generality, it is worth noting that even in the case of infinite shunt resistance, a relationship between $V_{o c}$ and light intensity could not be logarithmic across the entire range of irradiance, nor could $V_{o c}$ tend to minus infinity in the dark. This artefact simply results from the

This article is protected by copyright. All rights reserved. 
oversight of thermal charge carrier concentration in Koster's model. This irrelevant - in our case scenario is already reflected in the basic, ideal photodiode model which does describe a transition from logarithmic to linear regime but at irradiance and voltage levels much lower than observed here in this work:

$V_{o c}=V_{t} \cdot \ln \left(1+\frac{J_{p h}}{J_{s}}\right) \approx\left\{\begin{array}{c}V_{t} \cdot J_{p h} / J_{s}, J_{p h} \ll J_{s} \Leftrightarrow V_{o c} \ll V_{t} \\ V_{t} \cdot \ln \left(J_{p h} / J_{s}\right), J_{p h} \gg J_{s} \Leftrightarrow V_{o c} \gg V_{t},\end{array}\right.$

where $V_{t}=k_{B} T / e$ denotes the thermal voltage, $J_{S}$ the saturation current density, and $J_{p h}=e P G$ the flux of photo-generated charge carriers.

The non-infinite shunt resistance originates from various current losses in the photodetector including currentleakages from the edge of the device and/or due to the existence of pinholes and traps in the thin film ${ }^{[39]}$ As these parameters are yet to be controlled in organic devices, a small device-to-device variation in $R_{s h}$ is observed (Figure S16). Within the linear regime, each device will thus exhibit a slightly different voltage reading for a given value of the illumination (see Figure S17), as predicted by equation (4). Nevertheless, once the device is calibrated, and given the linear response, our approach can lead to an easy light detection in $V_{o c}$ mode by using a basic OPD device structure and simple instrumentation. The model in equation (4) also predicts that the higher the $E_{\text {gap }}$ is, the larger the range of linear responsivity is observed. This relation is illustrated in Figure S18. Organic photodetectors (or photovoltaic cells) featuring large $V_{o c}$ levels at 1 sun are thus more suitable for light detection in $V_{o c}$ mode. According to equations ( 3 ) and (4), the open-circuit voltage also strongly depends on temperature. The effect is greatly observed in organic solar cells where strong light intensity $\left(100 \mathrm{~mW} \mathrm{~cm}^{-2}\right)$ can significantly raise device temperature. ${ }^{[40]}$ This high temperature could influence the stability of voltage signals in OPDs. Nevertheless, as photodetectors operate mostly at low irradiance, we did not observe any heating of the photodiode and the OPD measurements are thus highly reproducible.

\subsection{Transient voltage response characteristics}

To further explore the OPD performance in $V_{o c}$ mode, we measured rise and fall times of the photovoltage signal. Figure 5 a displays the $V_{o c}$ response to a $50 \mathrm{~ms}$ light pulse with an incident power density of $25.1 \mu \mathrm{W} \mathrm{cm}{ }^{-2}$ at $536 \mathrm{~nm}$. The photodetector exhibits a $V_{o c}$ of $296 \mathrm{mV}$ with a rise time $t_{r}$ of $1.8 \mathrm{~ms}$ and a $V_{o c}$ fall time $t_{f}$ of $2.2 \mathrm{~ms}$. For comparison, the photocurrent response times are typically in the $\sim \mu$ s range for organic and perovskite photodetectors operating in current mode, ${ }^{[16,41]}$ and in the $\sim$ ms range for photomultiplication type organic photodetectors. ${ }^{[42,43]}$ One must note here that photovoltage and photocurrent responses differ in their nature. Nevertheless, even though only modest response times in $V_{o c}$ mode can be achieved (see Figure 5), this bandwidth is sufficient for many applications. ${ }^{[25]}$

This article is protected by copyright. All rights reserved. 

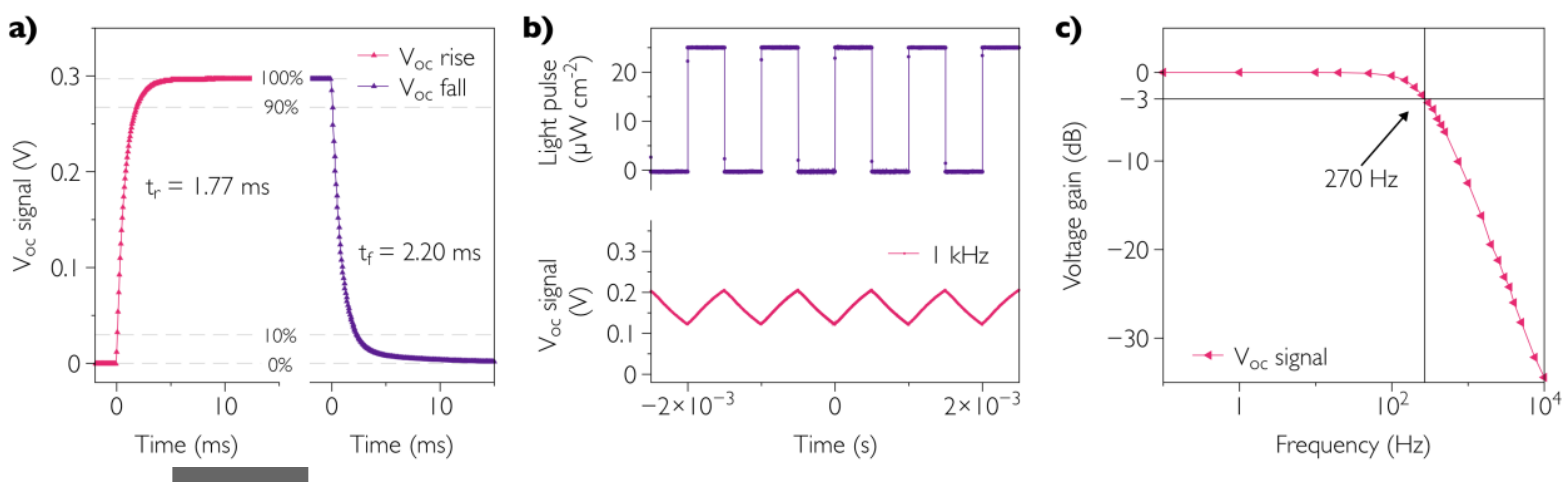

Figure 5. Transient voltage $\left(V_{o c}\right)$ performance of organic photodetectors. a) $V_{o c}$ response (rise and fall times) of the fabricated device to a $20 \mathrm{~ms}$ light pulse at $536 \mathrm{~nm}$ with an incident power density equal to $25.1 \mu \mathrm{W} \mathrm{cm}{ }^{-2}$. b) Transient $V_{o c}$ response at a pulse frequency of $1 \mathrm{kHz}$ (above the cut-off frequency). c) Voltage $\left(V_{o c}\right)$ gain expressed in $\mathrm{dB}$ as a function of increasing pulse frequency. The $-3 \mathrm{~dB}$ threshold corresponds to the frequency at which the $V_{o c}$ signal is attenuated by 1.414 .

The response time in voltage mode is further found to be influenced by light intensity (the brighter the light, the faster the signal) as shown in Figure S19. This finding is consistent with the notion of charge-density dependent recombination rates. ${ }^{[44-46]}$ More details can be found in Figure S20. As the pulse frequency increases, it is expected that the voltage signal will be attenuated. Detailed voltage measurements at low frequencies, from $1 \mathrm{~Hz}$ to $200 \mathrm{~Hz}$, and at high frequencies, from $500 \mathrm{~Hz}$ to 10 $\mathrm{kHz}$, can be seen in Figure $\mathrm{S} 21$ and $\mathrm{S} 22$, one particular case of $1 \mathrm{kHz}$ is shown in Figure $5 \mathrm{~b}$. One can note that the $V_{b c}$ signal is detectable despite the fact it does not reach its maximum ( $0.3 \mathrm{~V}$ ) nor minimum ( $\mathrm{QV}$ ). We consider this metastable mode as an exciting opportunity for OPDs to be used in high-frequency applications. The voltage gain as a function of frequency is shown in Figure $5 \mathrm{c}$. The cut-off frequency at $-3 \mathrm{~dB}$, i.e., at which the voltage signal is reduced by $\sqrt{2}$, is measured at $270 \mathrm{~Hz}$. The corresponding cut-off frequencies in the conventional photocurrent mode are 2 to 3 orders of magnitude higher. ${ }^{[15,17]}$ Nevertheless, this is still significantly higher than the human flicker fusion threshold $(\sim 60 \mathrm{~Hz}),{ }^{[47]}$ and one could find multiple commercial applications for such mode of operation including human-machine interfaces, motion detectors, scanners or camera sensors. One example of such practical applications is described in our recent study on optical pressure sensors where voltage readings are performed at $50 \mathrm{~Hz} .{ }^{[25]}$

\section{Conclusions}

\section{Traditionally, open-circuit voltage is the key element of photovoltaic cells. Here we have shown that} one can operate an organic photodetector in $V_{o c}$ mode to accurately sense low light intensities. The proposed approach offers a number of advantages including high responsivity and large linear range. In particular, for basic-structure OPDs, the $V_{o c}$ mode presents an exciting workaround to avoid high dark currents and their role in reducing the OPD performance. As demonstrated in this work, a device strongly affected by dark currents in photocurrent mode is a highly sensitive sensor capable of detecting light intensities as low as $400 \mathrm{pW} \mathrm{cm}^{-2}$ while operating in $V_{o c}$ mode. The photovoltage detection can be achieved using simple laboratory instrumentation.

This article is protected by copyright. All rights reserved. 
Our work gives guidelines to further improve the performance of the OPD in photovoltage mode. Enlarged shunt resistance, for example, would enhance the responsivity of OPD at low light intensities. At the same time, it would yet impede the overall linear range of the detector in photovoltage mode, as seen by comparing the magnitudes of the exponential (responsible for the logarithmicregime), and linear (responsible for the linear regime) terms in equation (6). On the other hand, this comparison reveals that the $L D R$ can be increased by strategies that would likewise increase the $V_{o c}$ in the logarithmic regime, i.e. by improving the charge carrier dissociation probability $P$, reducing bimolecular recombination coefficient $\gamma$, or selecting materials with larger energy level difference $E_{\text {gap }}$. For this reason, we have selectively chosen OPDs based on rubrene and $C_{60}$ that feature large $V_{o c}$ of $940 \mathrm{mV}$ under solar simulator. One clear impact of this work would be to designa photodetector with even higher $V_{\text {oc }}$ levels to further improve the $L D R$. To achieve this, the photodetector device structure could be greatly simplified since the influence of dark current is eliminated More interestingly, a high- $V_{\text {oc }}$ organic photovoltaic cell could be used here for photosensing.

Altogether, the $V_{o c}$ mode provides new options for simple detection of low irradiance. It can be seen as a complimentary extension of the conventional photocurrent mode supplying a robust photosensing tool for potential commercial applications.

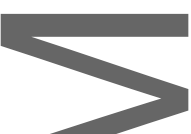

\section{Experimental Section}

Materials. PEDOT:PSS aqueous dispersion (1.3-1.7\%) was purchased from Heraeus and used without any furtherpurification. Rubrene (purity $99.99 \%$, sublimed grade), fullerene $\left(C_{60}\right.$, purity $99.5 \%$ ), barium (purity $99.9 \%$ ) and silver (purity 99.9\%) were purchased from Sigma Aldrich and used as received.

Device fabrication. The indium tin oxide (ITO) coated glass substrates (10 $\Omega$ per square, squares of $6.25 \mathrm{~cm}^{2}$ ) were first cleaned in deionized water with a detergent (Alconox from Sigma Aldrich), then sequentially cleaned in an ultrasonic bath with deionized water, acetone, ethanol and isopropanol (10 min each) and dried first under oxygen flow, then at $110^{\circ} \mathrm{C}$ for $5 \mathrm{~min}$. For the deposition process, PEDOT:PSS solution $(30 \mathrm{~nm}$ ) was spin-coated at 5,000 rpm for $60 \mathrm{~s}$ on the ITO substrates. Samples were then dried at $120^{\circ} \mathrm{C}$ for $10 \mathrm{~min}$ in air and moved into a nitrogen-filled glovebox for the thermal evaporation chamber. Rubrene $(35 \mathrm{~nm})$, fullerene $(25 \mathrm{~nm})$, barium $(10 \mathrm{~nm})$ and silver $(60 \mathrm{~nm})$ were then evaporated at slow rate $\left(<0.02 \mathrm{~nm} \mathrm{~s}^{-1}\right)$ and under high vacuum $\left(10^{-6} \mathrm{mbar}\right)$. The devices were post-annealed in the glovebox for $30 \mathrm{~min}$ at $80^{\circ} \mathrm{C}$ prior to testing. The device active area was 2.25 $\mathrm{mm}^{2}$. 


\section{WILEY-VCH}

Device characterization. Current density-voltage $(J-V)$ characteristics, open-circuit voltage $\left(V_{o c}\right)$ and linearity $(L D R)$ measurements were recorded by using a high power, green (528 nm) LED (ILH-ONO4TRGR-SC201) supplied by Intelligent LED Solutions (ILS) and calibrated with a silicon diode (Osram BPX 61).A similar LED (ILH-ONO1-TRGR-SC201-WIR200 from ILS) was used for transient measurements. Two ND filters (Thorlabs) were used: NE52OB-A (OD: 2) and NE550B-A (OD:5). USB4000 spectrometer from Ocean Optics was used to acquire electroluminescence spectrum of the LED. A Keithley 2604B dual channel source measure unit was used to power the LED and record data from organic photodiodes. A Keysight oscilloscope (InfiniiVision DSOX2004a) and an Agilent function generator $(33500 B)$ were both used for transient measurements. An Agilent digital multimeter (34450A) was used to record low voltage measurements. A Newport quantum efficiency measurement solution (QUANTX-300) was used to measure the external quantum efficiency and resulting responsivity. Optoelectrical characterization of diodes was carried out in a double shielded box (Faraday cage). All measurements were performed in air with the device being exposed to nitrogen flow.LabVIEW scripts were written to acquire and interpret different input signals remotely.

Supporting Information

Supporting Information is available from the Wiley Online Library or from the author.

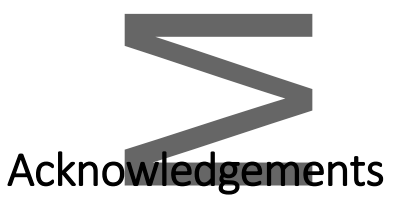

The joint research program between Intelligent Bionics and Soft Robotics at Queensland University of Technology (QUT) and Queensland Brain Institute at the University of Queensland funded this research. This work has also been partially funded by Australia India Strategic Research Fund (Project AISRF53820) and by the French National Research Agency (ANR) through the TAPIR project $N^{\circ}$ ANR15-CE24-0024-01. We also acknowledge Central Analytical Research Facility (CARF) at QUT for use of experimental facilities.

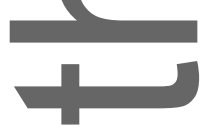

Conflict of Interest

The authors declare no conflict of interest.

This article is protected by copyright. All rights reserved. 
Received: ((will be filled in by the editorial staff))

Revised: ((will be filled in by the editorial staff))

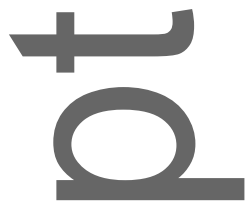

Published online: ((will be filled in by the editorial staff))

\section{References}

[1] G. J.Hedley, A. Ruseckas, I. D. W. Samuel, Chem. Rev. 2017, 117, 796.

[2] C. Park, K. Cho, D. H. Sin, H. Lee, J. H. Park, Adv. Mater. 2018, 30, 1800453.

[3] O. Inganäs, Adv. Mater. 2018, 30, 1800388.

[4] O. Ostroverkhova, Chem. Rev. 2016, 116, 13279.

[5] X. Liu, Y. Lin, Y. Liao, J. Wu, Y. Zheng, J. Mater. Chem. C 2018, 6, 3499.

[6] J. Lee, P. Jadhav, M. A. Baldo, Appl. Phys. Lett. 2009, 95, 033301.

[7] L. Li, F. Zhang, W. Wang, Q. An, J. Wang, Q. Sun, M. Zhang, ACS Appl. Mater. Interfaces 2015, 7,5890

[8] X. Liu, H. Wang, T. Yang, W. Zhang, X. Gong, ACS Appl. Mater. Interfaces 2012, 4, 3701.

[9] M. Gao, Y. Lin, F. Zhang, W. Wang, J. Miao, J. Zhang, L. Li, X. Zhan, Adv. Opt. Mater. 2016, 4, 1711.

[10] K. M. Sim, S. Yoon, J. Cho, M. S. Jang, D. S. Chung, ACS Appl. Mater. Interfaces 2018, 10, 8405.

[11] R. D. Jansen-Van Vuuren, A. Pivrikas, A. K. Pandey, P. L. Burn, J. Mater. Chem. C 2013, 1, 3532.

[12] D. Yang, X.Zhou, D. Ma, A. Vadim, T. Ahamad, S. M. Alshehri, Mater. Horizons 2018, 5, 874.

[13] Q. Song, T. Lin, Z. Su, B. Chu, H. Yang, W. Li, C. S. Lee, J. Phys. Chem. Lett. 2018, 9, 6818.

[14] J. Miao, F. Zhang, Laser Photonics Rev. 2018, 13, 1800204.

[15] R. D. Jansen-van Vuuren, A. Armin, A. K. Pandey, P. L. Burn, P. Meredith, Adv. Mater. 2016, 28,4766 .

[16] M. Kielar, O. Dhez, G. Pecastaings, A. Curutchet, L. Hirsch, Sci. Rep. 2016, 6, 39201.

[17] K.-J. Baeg, M. Binda, D. Natali, M. Caironi, Y. Noh, Adv. Mater. 2013, 25, 4267.

[18] S. Bin Lim, C. H. Ji, I. S. Oh, S. Y. Oh, J. Mater. Chem. C 2016, 4, 4920.

[19] D. S. Leem, K. H. Lee, Y. N. Kwon, D. J. Yun, K. B. Park, S. J. Lim, K. S. Kim, Y. W. Jin, S. Lee, Org.

This article is protected by copyright. All rights reserved. 
Electron. physics, Mater. Appl. 2015, 24, 176.

[20] D. J. Starkenburg, P. M. Johns, J. E. Baciak, J. C. Nino, J. Xue, J. Appl. Phys. 2017, 122.

[21] S. Xiong, L. Li, F. Qin, L. Mao, B. Luo, Y. Jiang, Z. Li, J. Huang, Y. Zhou, ACS Appl. Mater. Interfaces 2017, 9, 9176.

[22] Y. Fang, A. Armin, P. Meredith, J. Huang, Nat. Photonics 2019, 13, 1.

[23] A. K. Pandey, J. M. Nunzi, Adv. Mater. 2007, 19, 3613.

[24] A. K. Pandey, Sci. Rep. 2015, 5, 7787.

[25] M. Kielar, T. Hamid, L. Wu, F. Windels, P. Sah, A. K. Pandey, ACS Appl. Mater. Interfaces 2019, $11,21775$.

[26] F. Stöckmann, Appl. Phys. 1975, 7, 1.

[27] P. Irkhin, A. Ryasnyanskiy, M. Koehler, I. Biaggio, Phys. Rev. B - Condens. Matter Mater. Phys. 2012, 86, 085143.

[28] W. .. Potscavage, S. Yoo, B. Kippelen, Appl. Phys. Lett. 2008, 93, 193308.

[29] M. Kielar, M. Daanoune, O. François-Martin, B. Flament, O. Dhez, A. K. Pandey, S. Chambon, R. Clerc, L. Hirsch, Adv. Electron. Mater. 2018, 4, 1700526.

[30] V. V.Brus, Org. Electron. physics, Mater. Appl. 2016, 29, 1.

[31] N.K.Elumalai, A. Uddin, Energy Environ. Sci. 2016, 9, 391.

[32] Y. Zhang, X. D. Dang, M. Kuik, S. R. Cowan, P. Zalar, C. Kim, T. Q. Nguyen, Energy Environ. Sci. 2013, 6, 1766.

[33] H. J. Snaith, L. Schmidt-Mende, M. Grätzel, M. Chiesa, Phys. Rev. B - Condens. Matter Mater. Phys. 2006, 74, 045306.

[34] L. J. A. Koster, V. D. Mihailetchi, R. Ramaker, P. W. M. Blom, Appl. Phys. Lett. 2005, 86, 123509.

[35] A. K.Thakur, H. Baboz, G. Wantz, J. Hodgkiss, L. Hirsch, J. Appl. Phys. 2012, 112, 044502.

[36] C. M. Proctor, T. Q. Nguyen, Appl. Phys. Lett. 2015, 106, 083301.

[37] S. R. Cowan, W. L. Leong, N. Banerji, G. Dennler, A. J. Heeger, Adv. Funct. Mater. 2011, 21, 3083.

[38] M. M. Mandoc, F. B. Kooistra, J. C. Hummelen, B. de Boer, P. W. M. Blom, Appl. Phys. Lett. 2007, 91, 263505.

This article is protected by copyright. All rights reserved. 


\section{WILEY-VCH}

[39] B. Qi, J. Wang, Phys. Chem. Chem. Phys. 2013, 15, 8972.

[40] G. Chen, C. Si, Z. Tang, K. Guo, T. Wang, J. Zhang, B. Wei, Synth. Met. 2016, 222, 293.

[41] H. Liu, X. Zhang, L. Zhang, Z. Yin, D. Wang, J. Meng, Q. Jiang, Y. Wang, J. You, J. Mater. Chem. C 2017, 5, 6115.

[42] R. Nie, X. Deng, L. Feng, G. Hu, Y. Wang, G. Yu, J. Xu, Small 2017, 13, 1603260.

[43] J. W. Lee, D. Y. Kim, F. So, Adv. Funct. Mater. 2015, 25, 1233.

[44] A. V. Nenashev, M. Wiemer, A. V. Dvurechenskii, L. V. Kulik, A. B. Pevtsov, F. Gebhard, M. Koch, S. D. Baranovskii, Phys. Rev. B 2017, 95, 104207.

[45] M. Tachiya, K. Seki, Phys. Rev. B 2010, 82, 085201.

[46] G. Lakhwani, A. Rao, R. H. Friend, Annu. Rev. Phys. Chem. 2014, 65, 557.

[47] H. de Lange Dzn, J. Opt. Soc. Am. 2008, 44, 380.

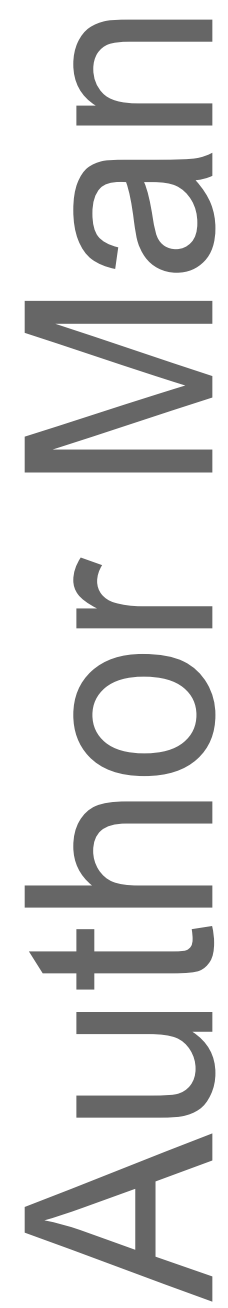

This article is protected by copyright. All rights reserved. 


\section{WILEY-VCH}

Table of Contents

The open-circuit voltage regime of organic photodetectors is revealed as a powerful method for detecting low light signals. The photovoltage light sensitivity across 9 orders of magnitude is demonstrated. This opens up new perspectives for resolving low light signals and provides simplified design rules for OPDs.

Keyword: Open-circuit voltage

Marcin Kielar , Tasnuva Hamid, Martin Wiemer, François Windels, Lionel Hirsch*, Pankaj Sah and Ajay K. Pandey

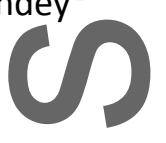

Light Detection in Open-circuit Voltage Mode of Organic Photodetectors
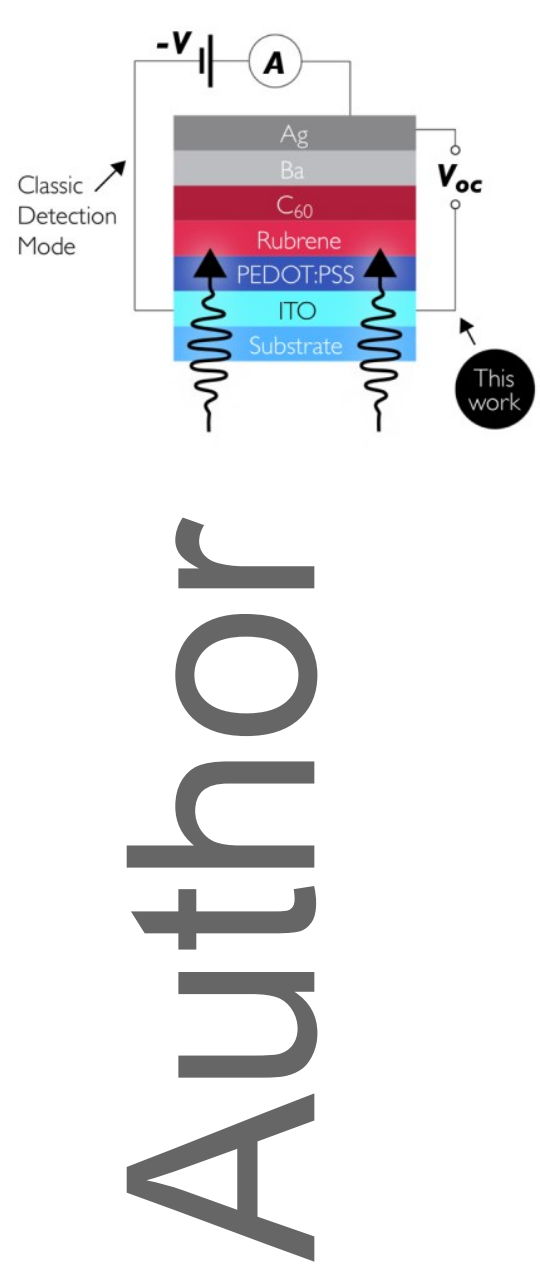

This article is protected by copyright. All rights reserved. 


\section{University Library}

\section{- M M N E R VA A gateway to Melbourne's research publications}

Minerva Access is the Institutional Repository of The University of Melbourne

\section{Author/s:}

Kielar, M;Hamid, T;Wiemer, M;Windels, F;Hirsch, L;Sah, P;Pandey, AK

Title:

Light Detection in Open-Circuit Voltage Mode of Organic Photodetectors

Date:

2020-02-01

Citation:

Kielar, M., Hamid, T., Wiemer, M., Windels, F., Hirsch, L., Sah, P. \& Pandey, A. K. (2020).

Light Detection in Open-Circuit Voltage Mode of Organic Photodetectors. Advanced

Functional Materials, 30 (9), pp.1907964-1907964. https://doi.org/10.1002/adfm.201907964.

Persistent Link:

http://hdl.handle.net/11343/285241 